\title{
DMEFS Web Portal: A METOC Application
}

\author{
Avichal Mehra and Jim Corbin \\ Integrated Data Systems Laboratory \\ Engineering Research Center \\ Mississippi State University \\ Stennis Space Center \\ MS 39529 \\ Mehra@erc.msstate.edu
}

\begin{abstract}
Distributed Marine Environment Forecast System (DMEFS) is a research testbed for demonstrating the integration of various technologies and components prior to operational use as well as a framework in which to operate validated meteorological and oceanic (METOC) numerical models. The focus of the DMEFS is to create an open computational web portal for a distributed system for describing and predicting the marine environment that will accelerate the evolution of timely and accurate forecasting. The primary goals are to first, focus the adaptation of distributed (scalable) computational technology into oceanic and meteorological predictions, and secondly, to shorten the model development time by expanding the collaboration among the model developers, the software engineering community and the operational end-users. The web portal provides a secure, seamless access to high performance resources, hiding their complexity. It is extensible and is designed for rapid prototyping, validation and deployment of legacy computational models as well as new models and tools by providing a set of common toolkits.
\end{abstract}

\section{Background}

Oceanic prediction in the littoral is vastly more complex than in the open ocean. The temporal and special scales of variability are much shorter; the parameters of interest, such as waves, storm surge, optical clarity, tide, sediment transport, beach trafficability, currents, temperature, salinity, etc., are very different. In addition, the real time or even historic observations of these parameters, in many areas of potential interest, are either very restricted or limited at best. The ability to simulate the littoral environment rapidly, accurately, and across many temporal and spatial scales poses very significant challenges to gathering, processing, and disseminating information. At the same time, underlying computer architectures have undergone an equally drastic change. No longer in the business of making vector supercomputers, U.S. computer manufacturers produce multiprocessor computers made from commodity processors with a high-speed interconnect. While such architectures offer vast improvements in cost versus performance, software written for vector supercomputers must be extensively rewritten to run on these new architectures. Thus, there is an 
emerging demand for scalable parallel models that not only can run on current architectures, but also can be easily adapted to new ones [1]. Finally, the exponential growth in data communications, exemplified by the Internet infrastructure and web applications, are enabling dramatic advances in distributed computational environments.

The Distributed Marine-Environment Forecast System (DMEFS) [2] attempts to address three essential goals. First, it incorporates the latest advances in distributed computing. Second, it provides the means of substantially reducing the time to develop, prototype, test, and validate simulation models. Third, it supports genuine, synergistic collaboration among computer specialists, model developers, and operational users. Several critical components that such a system must have to advance these three goals are discussed in this paper.

\section{Importance of DMEFS to the Navy}

Currently, most models are developed within the R\&D community as stove-pipe systems with unique requirements for control of execution, inputs and outputs. All of which are accomplished by unique execution of scripts that usually function properly only in the original research environment within which they were developed. When the model is transitioned to the operational community, the scripts have to be rewritten to accommodate the new operating environment, new data inputs, and new product outputs. As a result, many man-hours are needed just to get the new model to function in the new operating environment.

DMEFS provides a virtual common computer operating environment within which to both develop the new models and to operate them once validated. Secondly, as new models have been introduced into the operational community, each one usually uses unique applications to process input and output data, such as quality control, data interpolation schemes, and graphics. Therefore an operator must know several different ways to do the same task depending on the model of interest. Within DMEFS, from the user's perspective, the same functions will be done the same way through a common graphical user interface. Thus enormous time is saved in training and indoctrination of new personnel or when introducing a new model. Thirdly, in the current way of doing business, when a program sponsor needs updated information regarding a project or status of model, he must ask for input from the developer or an operator. With the web portal access of DMEFS, any authorized user can come directly into the system to obtain information and graphics. And finally, while most computer software developments within the Navy research community are beginning to use industry standards, most METOC model development does not. Developing new models within the DMEFS infrastructure allows the model developers to exploit the latest in industry standard software engineering technology without significant changes to their underlying code. 


\section{DMEFS Web Portal}

The DMEFS web portal targets the creation of an open, extensible framework, designed for rapid prototyping, validation, and deployment of new models and tools. It is operational over evolving heterogeneous platforms distributed over wide areas with web-based access for METOC tools and for forecast-derived information. The open framework is critical to facilitate the collaboration among the researchers within the community.

DMEFS web portal is a "meta-system" that provides the model developer with desktop access to geographically distributed high performance computational resources, both hardware and software. The portal is developed using enabling high performance and commodity technologies and services. It provides the foundation to facilitate the rapid development, integration, testing, and deployment of various meteorology-oceanography meta-applications. It simplifies the applications programming environment and nurtures a collaborative environment for users in a virtual workplace. The resources owned and managed by the stakeholders are made available in a controlled and secured manner.

\section{DMEFS Portal Services}

The DMEFS is a service oriented architecture to support high performance distributed computing.. Any METOC application embedded in DMEFS or developed using DMEFS may also take advantage of the various services provided by DMEFS.

A set of services provided by the DMEFS Web Portal currently under development are (see Figure 1):

Metacomputing Services: These services include a spectrum of high performance scalable computers (supercomputers) with sufficient computational power to perform the required calculations and an extensive set of software tools to aid in model development. These computers may be distributed across a local area network and/or a wide area network. The computational facilities include experimental and operational high speed networks, digital libraries, hierarchical storage devices, visualization hardware, etc. It is essential to provide services to facilitate their discovery and use. Security and access control services enable the resource providers to offer the use of the resources in a controlled manner using a flexible access control policy. Resource management services allow the registration and scheduling the availability of the resources in a metacomputing environment. A few preliminary glimpses of such an environment with screen-shots are presented in [3].

METOC Model Services: METOC Model Services is a suite of realistic numerical oceanic and atmospheric models optimized for the new scalable computer architectures, showing useful forecast skill on time and space scales of scientific interest. A suite also consists of model test cases and skill assessment procedures for model validation, comparison and testing.. Since the most skillful forecasts are made by coupled model-observation systems with data assimilation and real-time adaptive sampling, a forum for testing methods for assimilation of the mesoscale in situ and remote observations including radar observations will also be provided in future. Some other issues which can be studied include treatment of open boundary conditions including multi-scale nesting. Interfaces to the METOC Model Services enables the scheduling, execution, and validation of the model results. 


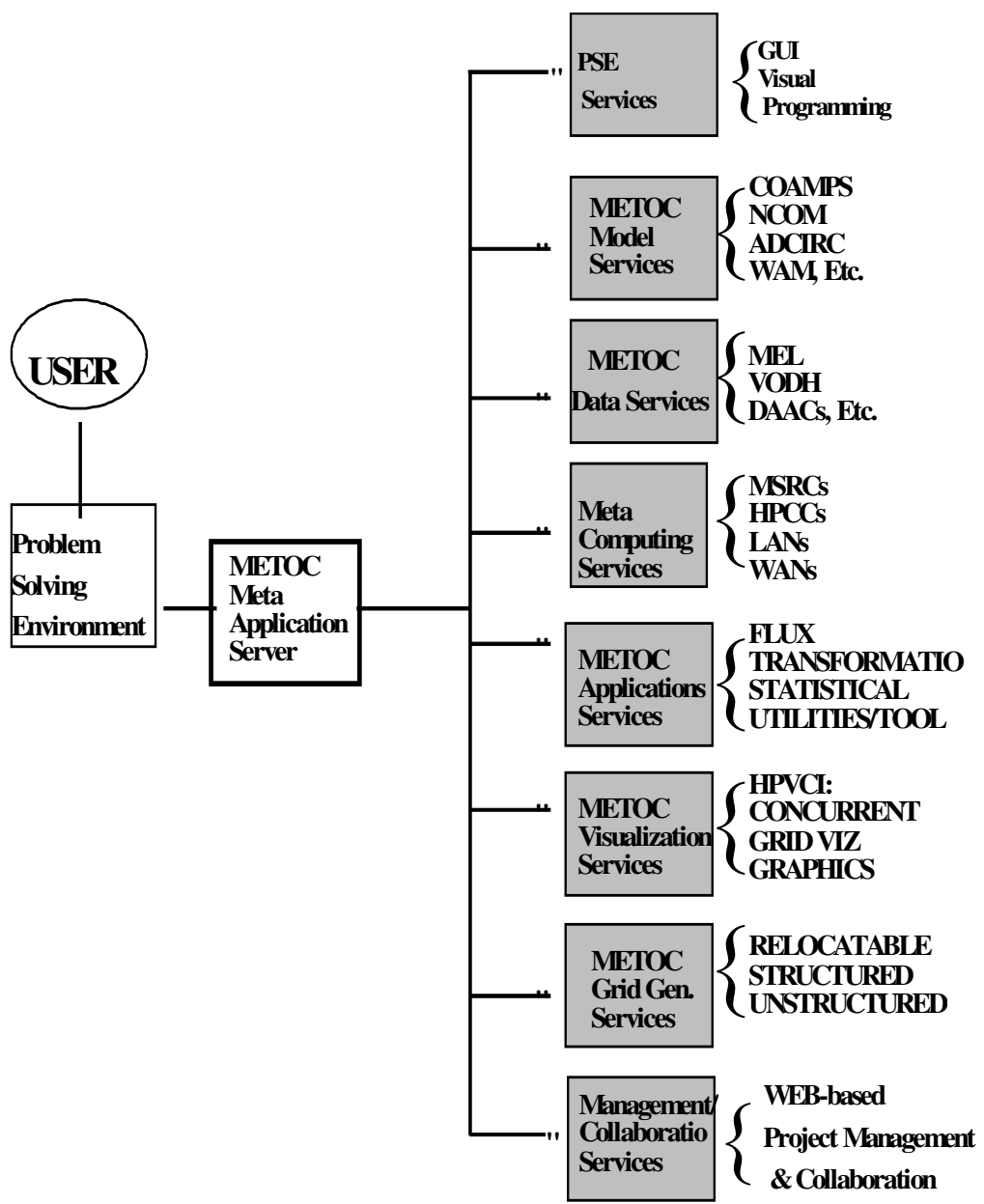

Fig. 1. DMEFS Portal Services

\section{DMEFS Portal Services (Cont.)}

A few more services to be provided by DMEFS web portal in the future are:

Problem Solving Environment (PSE): The DMEFS PSE will provide a visual programming paradigm similar to that employed by a number of scientific visualization software such as AVS, SGI/Explore, KHOROS, etc. It will allow the scientists and engineers to work comfortably and intuitively in an environment that allows the users to set up their task and data flow modules and connect them via data pipes to compose and execute applications. This model and/or the associated visual programming paradigm has been adopted by other metacomputing initiatives. The PSE in DMEFS will allow the users to authenticate their identity, discover the availability of numerical models and couple them appropriately; discover, acquire, and preprocess appropriate initialization and assimilation data; set-up and execute the 
models on appropriate hardware and postprocess the results. A scripting mechanism will allow the user to store and execute a set of routinely exercised tasks.

METOC Data Services: Numerical models require input data for initialization, boundary conditions and up-date (assimilation). These data may be from a number of sources including observational platforms (including in-situ and remotely sensed), and other numerical models and applications. These (data assimilative) models also generate large volumes of data that need to be managed, analyzed, and distributed. An environmental forecast system also requires the production of useful products that assist in the decision making process. In a distributed computational environment these datasets are usually stored in repositories that are geographically distributed on heterogeneous data management systems employing proprietary and special formats. A seamless mechanism is required for discovering the availability of these datasets, determining their quality, and transparently accessing them. Moreover, these datasets and products need to be published in the digital library and their availability advertised. Further, in an interoperable environment interface specifications need to be evolved and implemented for transparent access and use of these datasets from the various repositories.

METOC Visualization Services: DMEFS will address the fundamental issues of remote visualization and the management/manipulation of very large geophysical and simulation datasets. The visual programming metaphor will provide the researcher with access to the METOC Visualization Services. Examples of visualization services required include the following:

- $\quad$ visualization tools to browse geography and climate/observation databases

- grid visualization tools (in the absence of any solution data) to aid in debugging grids and models under development; such tools should also provide automated grid quality diagnostics and the ability to compare grids

- concurrent model visualization tools; while these should certainly include visualizations of solutions in progress, there would also be substantial value in providing visualization tools to examine the run-time performance of models (e.g., concurrent visualization of processor loads).

With distributed concurrent visualization, the user will be able to set up and run a simulation in a remote computing environment and then utilize the equipment in the remote compute environment to do part of the visualization task, potentially concurrently.

METOC Grid Generation Services: DEMFS will develop a robust, economical, and efficient structured/unstructured grid generation system tailored to support ocean and atmospheric models within the DTA framework. The METOC Grid Generation Services will support the generation of quality 2-D, 2.5-D and 3-D structured/unstructured grids. It will seamlessly obtain geometric and ocean characteristics by interrogating the DMEFS METOC Data Services.

Application Analysis Services: A spectrum of utilities and tools is required to extract information from the very large geophysical and simulation datasets for various reasons (e.g., data compression for transmission and storage, feature extraction for data steering and adaptation, spatial and time scaling or transformation for coupling different applications, and extract statistical characteristics of results). As an example, an analysis process may be run "adjacent" to a compute intensive storm surge simulation, perhaps concurrently, to extract information of the response (compressed and scalable in an appropriate "metacomputing" format) for 
transmission, storage, and subsequent transformation to the specific requirements for a receiving process for structural simulation at a distant location. Tools are also required to extract the macromodels for parametric simulations.

Management and Collaboration Services: Tools and data services are desirable for posting and reviewing program and project status, targeting two levels of access: internal for use by the DMEFS management and researchers and for appropriate external use by sponsors. A convenient WEB-based project management tool, which targets ease of use, is anticipated to provide means to quickly enter and modify goals at the individual and different organizational levels while providing appropriate access for cross-disciplinary research in a virtual organization. In addition, there is the need to establish real-time collaborative tools between remote researchers to facilitate hands-on demonstrations and communications (e.g., shared applications associated with desktop teleconferencing).

\section{DMEFS Portal Users}

Overall, three different groups of users of a fully functional DMEFS web portal can be identified. These are (as indicated in Figure 2) researchers, operators and managers.

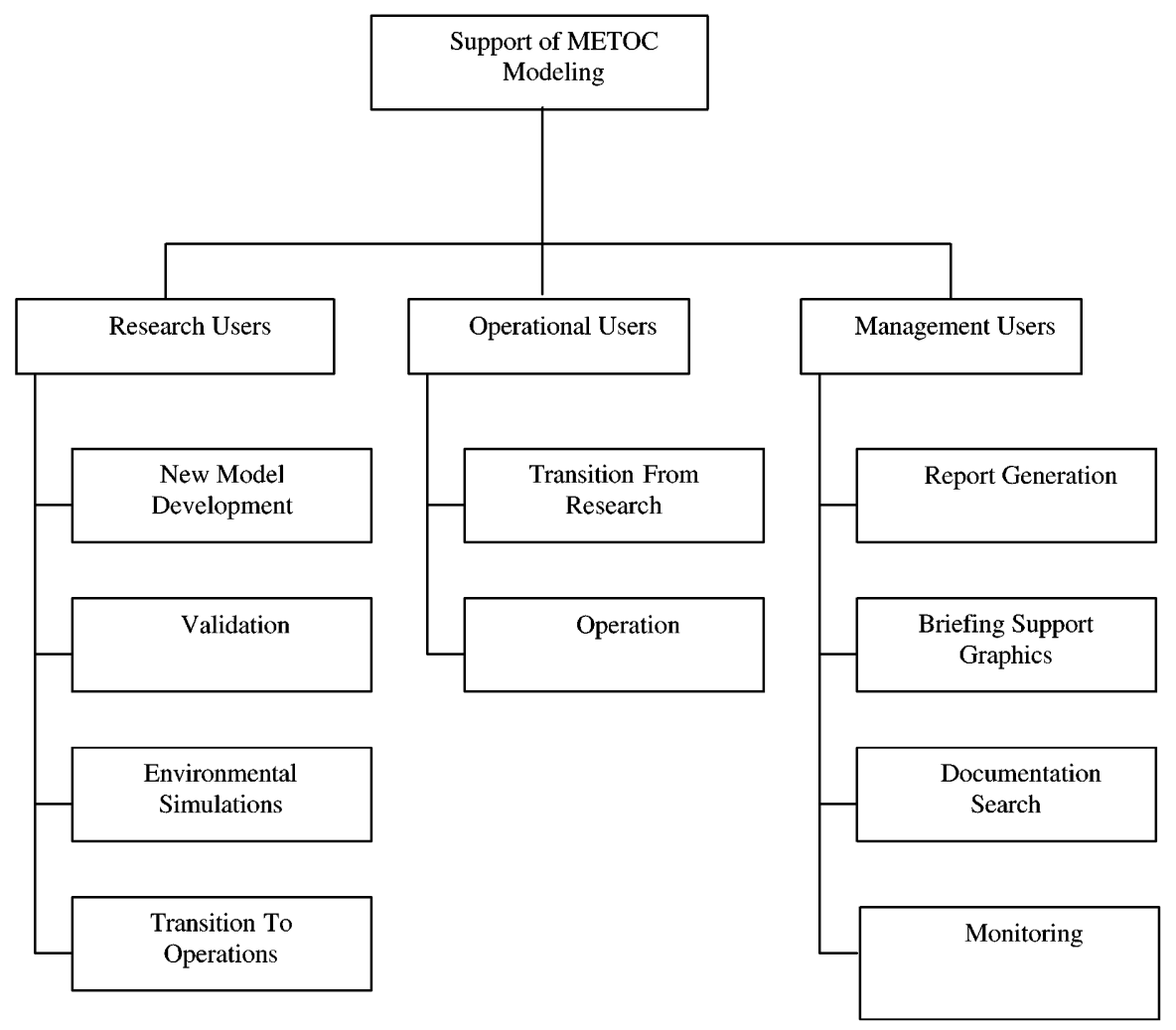

Fig. 2. High Level View of DMEFS Web Portal Users 
The high level view of the major tasks performed by the three classes of users is shown in Figure 2. Figures 3, 4 and 5 present a further breakdown of those high level tasks in sub-tasks or objectives from each user perspective. The research support functions require the most flexibility and demand for real time system configuration. The basic tasks for a research user can be broadly defined under four categories: new model development, transition to operations, environmental simulations and validation. These tasks can be broken down into further sub-tasks as shown in Figure 3. The operational user is more focused on product generation and less on processes, but needs more automation and tailored environment for routine run streams for both operations (to run a model or to derive products from archived model runs) and transitions. These activities lead to other sub-tasks as shown in Figure 4.

The management user focus's more on monitoring status. He or she can be from research or operations, but can also represent project, program or sponsor management, or users from the public domain. These users are distinctly high-level users of the portal and would use web access primarily for information only. This information could be used for generating reports, making briefs, monitoring the status of the project and/or searching for relevant documents and references.

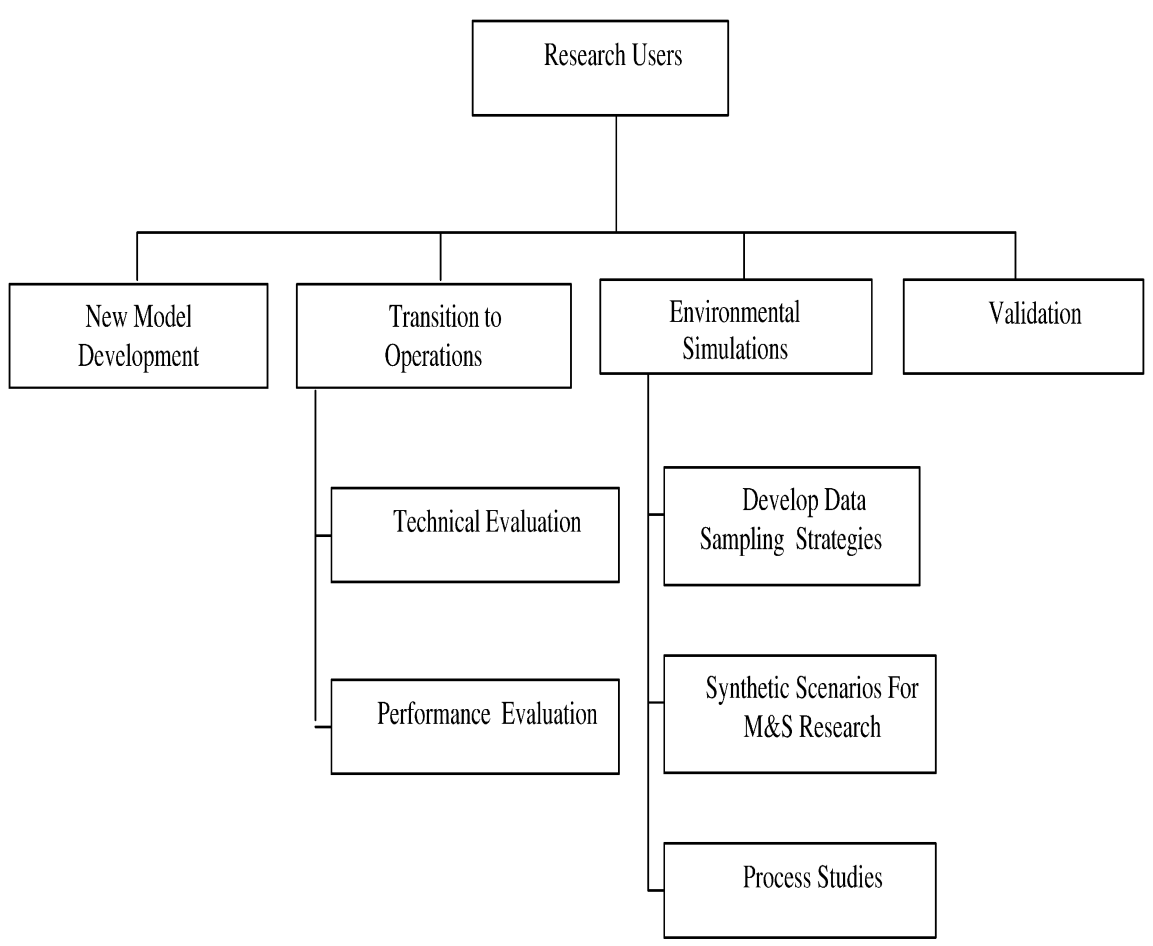

Fig. 3. Research Users for DMEFS Web Portal 


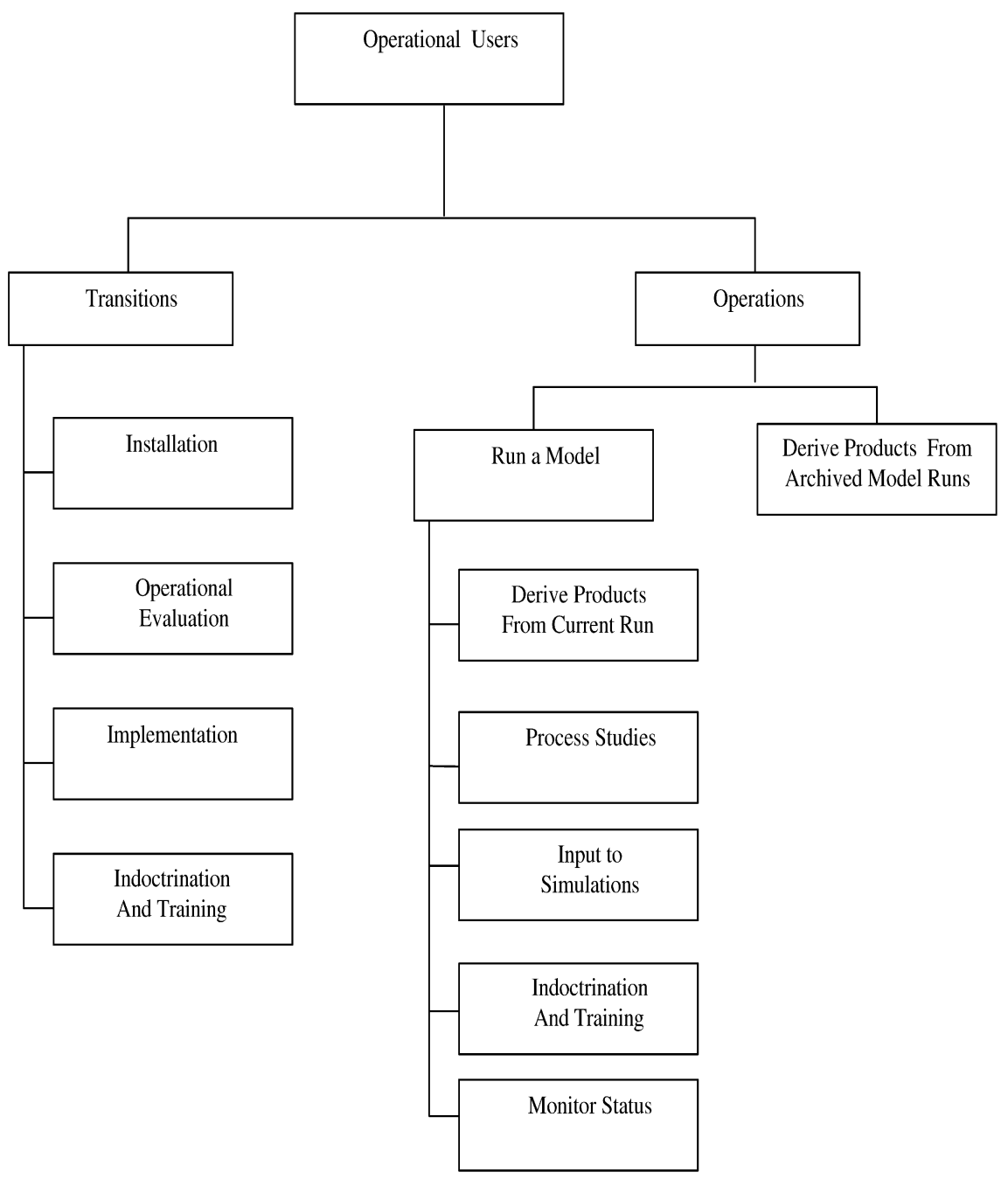

Fig. 4. Operational Users of DMEFS Web Portal 


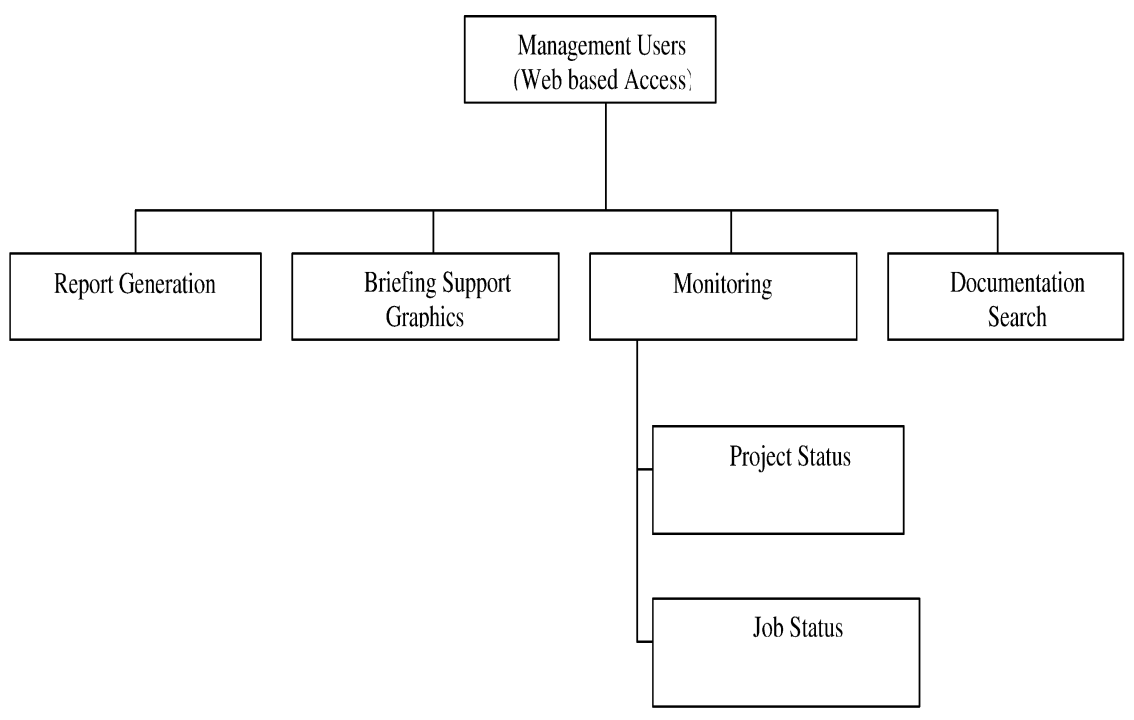

Fig. 5. Management Users of DMEFS Web Portal

\section{Summary}

The exponential growth in computing technologies has changed the landscape in high performance computing (HPC). The migration of HPC from vector supercomputers to both tightly-coupled shared-memory multiprocessors and loosely coupled distributed multiprocessors has complicated software development and maintenance of legacy codes. Second, the exponential growth in data communications, specifically the Internet infrastructure and web applications, are enabling dramatic advances related to distributed computational environments.

Distributed Marine-Environment Forecast System (DMEFS) web portal attempts to address the above two issues by providing a suite of services which are preconfigured for three varied classes of users namely the research, the operational and the management user typically found in the METOC community.

\section{References:}

1. Joseph W. McCaffrey, Donald L. Durham, and James K. Lewis, "A Vision for the Future of Naval Operational Oceanic Nowcast/Forecast Systems," Marine Technology Society Journal, Vol. 31, No. 3, pp. 83-84 (1997).

2. http://www.erc.msstate.edu/ haupt/DMEFS.

3. Tomasz Haupt, Purushotham Bangalore, and Gregory Henley, "A Computational Web Portal for the Distributed Marine Environment Forecast System," to be presented at the IEEE International Symposium on Cluster Computing and the Grid, 15-18 May, Brisbane Australia (2001). 